\title{
Roche plans for more convenient-to-use Herceptin and Rituxan
}

In January, the Swiss drug manufacturer Roche announced $\mathrm{a} € 190$ million (\$175 million) investment to manufacture a device that enables the subcutaneous injection of biologics, notably its blockbuster antibody cancer drug Herceptin (trastuzumab), by combining it with an enzyme that opens up channels in the extracellular matrix. San Diego-based Halozyme Therapeutics is supplying the enzyme, a recombinant hyaluronidase it has developed. The new Herceptin formulation is now in phase 3 clinical trials, and if the program were suc-

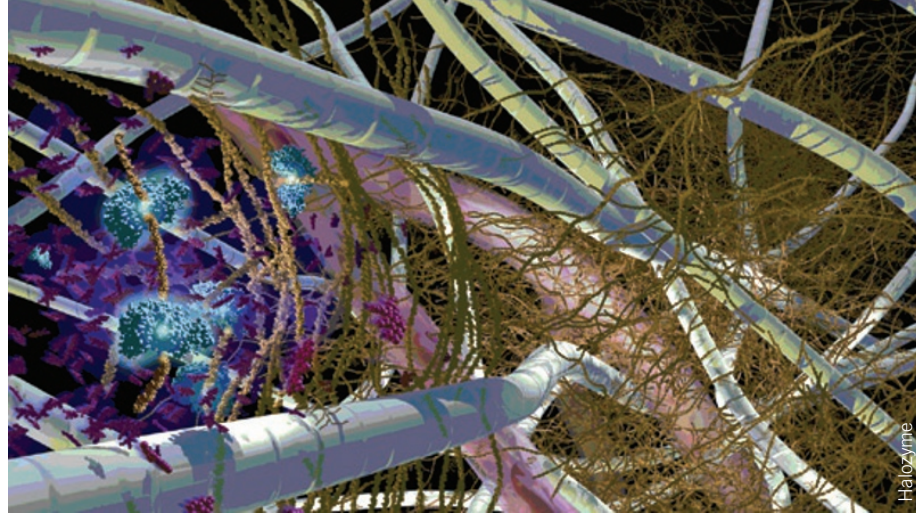

The extracellular matrix is temporarily broken down by Halozyme's human hyaluronidases, opening an opportunistic route to deliver biologics subcutaneously.

October, are expected toward the end of 2011, at which time Roche could seek marketing approval. "We can rely on the huge safety database of Herceptin and bridge to the Herceptin i.v. data," Schmidt says.

Herceptin was the most obvious candidate to establish proof of concept-especially for the part of the treatment where it is administered as monotherapy, Schmidt adds. "That is mainly the case in early breast cancer, once the patient has finished chemotherapy." Roche is also studying the efficacy of using Herceptin for two years instead of one, as cessful, it would offer patients a convenient dosing form of the drug, which is currently given via intravenous (i.v.) infusion. As a line extension, it expands the blockbuster drug franchise and extends the patent protection for Herceptin — a strategy Roche could apply to other biologics as well.

"When we looked at the Roche/Genentech portfolio of biologics, it became quite clear that, if we wanted a more convenient administration form, we needed tools that allow us to painlessly inject larger volumes," says Johannes Schmidt, project leader, biologics, for Roche in Basel. Roche completed its acquisition of Herceptin's developer, Genentech, in San Francisco, in 2009. Before that, the Swiss pharma owned part of the company and held marketing rights outside the US to Herceptin as well as other Genentech drugs.

Typically, the volume of a subcutaneous injection is limited to around $1 \mathrm{ml}$. Larger injection volumes are painful and cause tissue distortion, edema and irritation and redness (erythema). That's not an issue with some biologics, such as the tumor necrosis factor alpha inhibitors used to treat rheumatoid arthritis, where the volumes involved are very small. But the situation is different with cancer drugs. Herceptin, for example, is given at doses of $500 \mathrm{mg}$ or more. "A concentrated antibody formulation like 100 $\mathrm{mg} / \mathrm{ml}$ would take at least $5 \mathrm{ml}$ to inject someone," says Schmidt."That would not be possible without real pain."

Roche turned to Halozyme to solve the problem. The companies initiated a collaboration around recombinant human hyaluronidase (rHuPH20) in 2006. Hyaluronidases degrade hyaluronic acid (hyaluran) in the extracellular matrix, creating channels about $200 \mathrm{~nm}$ in diameter through which large molecules can pass to get into the bloodstream. "Most biologics, including monoclonal antibodies,

cytokines, recombinant enzymes, other proteins and peptides can be enabled by the technology," says Halozyme CEO Jonathan Lim. Bovine hyaluronidase has been in use for $>50$ years, mostly for ophthalmic surgery, where the enzyme facilitates local distribution of anesthetics. But repeat administrations of an animal-derived molecule cause immunogenicity, making it inappropriate for cancer maintenance therapy.

Recombinant HuPH20 suffers no such drawbacks, and it is also the only enzyme in the hyaluronidase family that works at neutral $\mathrm{pH}$ and has only temporary activity. "You only want to open the subcutaneous tissue to generate a cavity for a small period of time," says Schmidt."You don't want to leave gaps there." With rHuPH20, the gap is closed in 24 hours.

In addition to its collaboration with Roche, Halozyme has licensed rHuPH20 to Baxter Healthcare, in Northbrook, Illinois, for use in its Gammagard plasma-derived immune globulin. Halozyme is also developing a very fast-acting insulin by combining it with $\mathrm{rHuPH} 20$ to create a more physiologic pharmacokinetic profile that potentially allows for better glycemic control and potentially less weight gain. But Roche is not attempting to change the pharmacokinetics of its drugs using rHuPH20. Indeed, having a more convenient formulation otherwise comparable to the i.v. version helps speed the regulatory pathway.

Roche has brought three $\mathrm{rHuPH} 20$-formulated products into clinical trials with alreadyapproved drugs, including Herceptin, the leukemia and lymphoma antibody MabThera (rituximab, Rituxan in the US), and an undisclosed third candidate; the latter two are each in phase 1 safety studies. Initial results of the phase 3 Herceptin trial, which began dosing patients last maintenance therapy. "If two-year comes out to be superior, we have to have a more convenient regimen to support the patients," he says. Subcutaneous delivery would also have compliance benefits. As an infusion, "we have seen that patients don't even complete their one-year [program] sometimes," he says. "If they go to a clinic and see sick patients all around them, they may actually fall back, because in early breast cancer maintenance treatment they don't feel sick."

The drug is delivered subcutaneously as a single premixed solution containing $\mathrm{rHuPH} 20$, which is built into a glass cartridge that is packed into a motor-driven device. "The patient essentially only has to pull the cover of the adhesive off," says Schmidt. "Everything else is done automatically." The needle is inserted, the device injects the solution and the needle automatically retracts. In some countries (e.g., Germany), the device can be self-administered, whereas in others it will be used in the physician's office, depending on the country's needle requirements. The device would potentially work with any stable liquid formulation of a biologic. "Trastuzumab and rituximab are fairly stable antibodies," says Schmidt. “There may be others where that's an issue and we probably couldn't use our injection device."

Roche's production line in Kaiseraugst, Switzerland, will supply material for clinical studies and market launch of subcutaneous Herceptin, whereas its full-scale automated production line in Mannheim, Germany will deliver commercial supply to markets. Roche told investors in February that given the dynamics of the US oncology market, where physicians derive income from i.v. infusions, it is focusing on opportunities for subcutaneous delivery outside the US.

Mark Ratner Cambridge, Massachusetts 